\title{
BIOPROSPECÇÃO DA FLORA FÚNGICA ENDOFÍTICA DA RESERVA DE JURUBATIBA
}

\author{
Maria das Graças Machado Freire \\ Doutora em Biologia Molecular e \\ Funcional/Laboratório de Química e \\ Biomoléculas/ISECENSA/RJ (LAQUIBIO) \\ freire.mgm@gmail.com

\section{Vicente Mussi-Dias} \\ Doutor em Produção Vegetal/ Laboratório de \\ Química e Biomoléculas/ISECENSA/RJ \\ (LAQUIBIO) e Laboratório de Entomologia e \\ Fitopatologia/ CCTA/UENF/RJ \\ vimdias@yahoo.com.br
}

\author{
Hugo da Silva Coutinho \\ Técnico do LAQUIBIO \\ hugo_mrmt@hotmail.com \\ Gloria Andréia Ferreira Hernandez \\ Estagiária do LAQUIBIO \\ glorys97@hotmail.com \\ Luana Pinto de Souza Tavares \\ Estagiária do LAQUIBIO \\ luanatavares1996@gmail.com
}

\section{RESUMO}

A preservação do bioma Restinga é de grande importância, pois ao mesmo tempo que este se apresenta como um dos mais ameaçados de extinção, pouco se sabe sobre a diversidade de fungos endofíticos que ali habitam, uma vez que estes fungos podem ser potenciais produtores de substâncias para a indústria farmacêutica, de cosméticos e de alimentos. Para os nossos estudos foram coletadas 34 espécies vegetais no Parque Nacional da Restinga de Jurubatiba, sendo 27 identificadas até o momento: Blutaparon portulacoides, Commelina erecta, Ipomoea pes-caprae, Schinus terebinthifolius, Allagoptera arenaria, Smilax rufescens, Byrsonima sericea, Sideroxylon obtusifolium, Guapira opposita, Clusia hilariana, Eugenia astringens, Remirea marítima, Inga maritima, Eugenia uniflora, Erythroxylum ovalifolium, Myrsine parvifolia, Manilkara subsericea, Cupania emarginata, Tocoyena bullata, Ipomoea imperati, Centrosema virginianum, Capparis flexuosa, Jacquinia brasiliensis, Inga laurina, Myrsine guianensis, Protium heptaphyllum e Canavalia rosea. Destas plantas foram obtidos 400 fungos endofíticos pertencentes a diferentes gêneros: Exserohilum; Gliocladium; Pestalotiopsis; Cladosporium; Curvularia; Penicillium; Phoma; Trichoderma; Aspergillus; Bipolaris; Guignardia; Beauveria; Alternaria; Colletotrichum; Nigrospora; Fusarium e Phomopsis, dentre outros. Cada isolado fúngico encontra-se devidamente armazenado e depositado na micoteca do LAQUIBIO, a fim de se garantir a preservação destas espécies e o fornecimento de culturas viáveis desses fungos para utilização futura.

Palavras-chave: restinga, biodiversidade, fitoquímicos

\section{ABSTRACT}

Restinga forest biome preservation is of great importance because if on the one hand it is one of the most endangered biome in the world, for the other hand little is known about the diversity of its associated endophytic fungi, which may be potential producers of substances for the pharmaceutical, cosmetics and food. We collected 34 plant species in the Parque Nacional da Restinga de Jurubatiba and 27 were successfully identified until this moment: Blutaparon portulacoides, Commelina erecta, Ipomoea pescaprae, Schinus terebinthifolius, Allagoptera arenaria, Smilax rufescens, Byrsonima sericea, Sideroxylon 


\section{CENTRO EDUCACIONAL NOSSA SENHORA AUXILIADORA \\ INSTITUTOS SUPERIORES DE ENSINO DO CENSA CENTRO DE PESQUISA E PÓS-GRADUAÇÃO}

obtusifolium, Guapira opposita, Clusia hilariana, Eugenia astringens, Remirea marítima, Inga maritima, Eugenia uniflora, Erythroxylum ovalifolium, Myrsine parvifolia, Manilkara subsericea, Cupania emarginata, Tocoyena bullata, Ipomoea imperati, Centrosema virginianum, Capparis flexuosa, Jacquinia brasiliensis, Inga laurina, Myrsine guianensis, Protium heptaphyllum e Canavalia rosea. From these plants were obtained 400 endophytic fungi belonging to different genres: Exserohilum; Gliocladium; Pestalotiopsis; Cladosporium; Curvularia; Penicillium; Phoma; Trichoderma; Aspergillus; Bipolaris; Guignardia; Beauveria; Alternaria; Colletotrichum; Nigrospora; Fusarium e Phomopsis, among others. Each fungal isolate is properly stored and disposed in the LAQUIBIO mycology collection, in order to guarantee the preservation of these species and to supply viable cultures for future use of these fungi.

Key-words: restinga, biodiversity, phytochemical compounds

\section{1- INTRODUÇÃO}

Talvez não tenhamos hoje a real noção do que significou a criação do Parque Nacional da Restinga de Jurubatiba, diante da enorme diversidade que ali se encontra. Provavelmente, nas próximas décadas, após estudos mais detalhados dessa imensa riqueza, possamos avaliar o legado da criação desta reserva para as futuras gerações (Reis, 2006).

O Parque Nacional (PARNA Jurubatiba), localizado no nordeste do estado do Rio de Janeiro, conta com uma área litorânea de 14.860 hectares, abrangendo os municípios de Quissamã (65\%), Carapebus (34\%) e Macaé (1\%). Criado em 1998, é considerado Reserva da Biosfera pela UNESCO (Organização das Nações Unidas para a Educação Ciência e Cultura) (Vainer, 2010).

O PARNA de Jurubatiba que tem a maior área de restinga incluída em uma Unidade de Conservação do Estado do Rio de Janeiro conta com uma faixa de areia de $44 \mathrm{~km}$, com grande número de lagoas costeiras, o que fornece algo de beleza e fonte de exploração únicos (Luz et al., 2011). De acordo com a época do ano o número de lagoas pode variar de quatorze, quando as chuvas tornam-se escassas e algumas delas podem até secar (de maio a outubro), até dezoito no período de maior pluviosidade entre dezembro e maio (Campos, 2015).

A restinga é definida como planície arenosa costeira de origem marinha, abrangendo praias, cordões arenosos, dunas, depressões entre-cordões e depressões entre-dunas com respectivos brejos, charcos, alagados e lagoas, cuja vegetação e fauna estão adaptadas às condições ambientais locais (Decreto Estadual $\mathrm{n}^{\circ} 41.612$ de 2008).

No norte do Estado do Rio de Janeiro, na faixa mais próxima ao mar ainda existem alguns trechos de restinga preservados, destacando-se o Parque Nacional da Restinga de Jurubatiba (Reis, 2006) e a Reserva Particular do Patrimônio Natural (RPPN Fazenda Caruara) que com 3.845 hectares representa a maior unidade de conservação privada de restinga do Brasil abrigando um dos principais remanescentes preservados deste ecossistema (Freire et al., 2015).

Neste bioma cuja vegetação característica é consequente de uma combinação de fatores físicos e químicos, tais como elevada temperatura, salinidade, grande deposição de salsugem e alta exposição à luminosidade (Cogliatti-Carvalho et al., 2000), é crescente o interesse por micro-organismos endofíticos como fonte de bioativos naturais, ainda que as pesquisas sobre este tema, no bioma abordado, sejam inexpressivas até o momento. A busca por estas substâncias deve-se, em parte, ao fato de que microorganismos endofíticos são capazes de produzir compostos bioativos, os fitoquímicos, que são reconhecidamente produtos naturais sintetizados por suas plantas hospedeiras (Strobel et al., 2004). Além disso, os metabólitos produzidos pelos endófitos representam uma fonte enorme e inexplorada de estruturas

Persp. online: biol. \& saúde, Campos dos Goytacazes, 21 (6), 6-13, 2016

seer.perspectivasonline.com.br 


\section{CENTRO EDUCACIONAL NOSSA SENHORA AUXILIADORA \\ INSTITUTOS SUPERIORES DE ENSINO DO CENSA CENTRO DE PESQUISA E PÓS-GRADUAÇÃO}

químicas únicas que foram modificadas durante a evolução das espécies e em resposta às alterações em seus habitats, produzidas para a interação planta-endófito (Gunatilaka, 2006). Este trabalho teve como objetivo a exploração das comunidades de micro-organismos endofíticos de diferentes plantas nativas da Restinga Nacional de Jurubatiba, região norte do estado do Rio de Janeiro.

\section{2- MATERIAL E MÉTODOS}

\section{1- Área de estudo e coleta das amostras}

A coleta das plantas foi realizada em julho de 2015, numa área da restinga próxima à Lagoa de Jurubatiba, no Parque Nacional da Restinga de Jurubatiba. No presente trabalho, amostraram-se 34 espécies vegetais na zona de vegetação psamófila e halófila reptante (PHR), que se inicia próxima à escarpa praial, em faixa com largura de aproximadamente 5-10 m (Araujo et. al., 1998). A partir da zona PHR, foi realizada uma transecção perpendicular ao mar em direção ao interior da restinga, até alcançar áreas mais fechadas de moitas (Cogliatti-Carvalho et al., 2000), formando um retângulo $300 \mathrm{~m}$ x $150 \quad \mathrm{~m}$ (22 $2^{\circ} 17^{\prime} 53,30^{\prime \prime} \mathrm{S} / 41^{\circ} 41^{\prime} 13,59^{\prime \prime} \mathrm{O} ; \quad 22^{\circ} 17^{\prime} 48,38^{\prime \prime} \mathrm{S} / 41^{\circ} 40^{\prime} 05,58^{\prime \prime O}$; $\quad 22^{\circ} 17^{\prime} 44,22^{\prime \prime} \mathrm{S} / 41^{\circ} 40^{\prime} 08,20^{\prime \prime O}$; $\left.22^{\circ} 17^{\prime} 49,54^{\prime \prime} \mathrm{S} / 41^{\circ} 41^{\prime} 16,95^{\prime \prime} \mathrm{O}\right)$ com um dos lados margeando a Lagoa de Jurubatiba (Figura 1).

Coletaram-se amostras de folhas, as quais encontravam-se vivas, aderidas à planta no momento da coleta, sadias ou sem lesões aparentes. Cada espécie utilizada no isolamento dos fungos endofíticos foi comparada e identificada com base em espécies já depositadas no Herbário do Jardim Botânico do Rio de Janeiro.
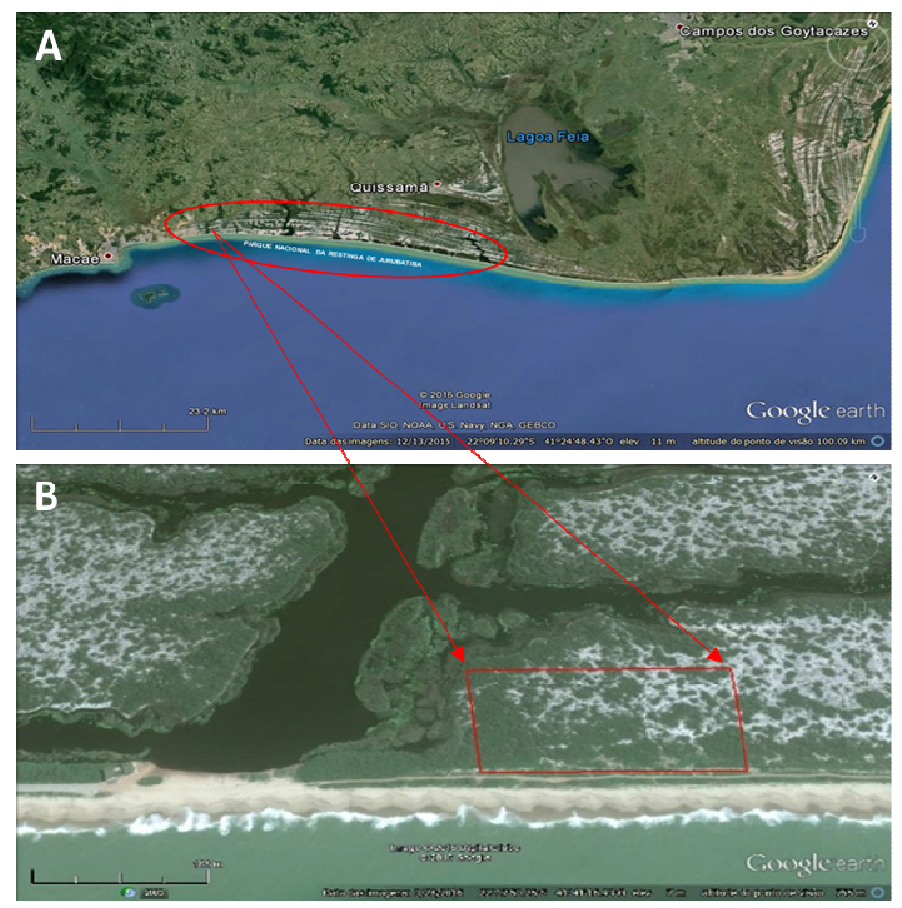

Figura 1. Parque Nacional da Restinga de Jurubatiba/RJ entre os municípios de Macaé e Quissamã/RJ. A) Vista aérea da localização da Restinga. B) Área próxima à Lagoa de Jurubatiba, cujas amostras vegetais foram coletadas para o isolamento de fungos endofíticos. 


\section{CENTRO EDUCACIONAL NOSSA SENHORA AUXILIADORA \\ INSTITUTOS SUPERIORES DE ENSINO DO CENSA \\ CENTRO DE PESQUISA E PÓS-GRADUAÇÃO}

\section{2- Desinfecção das amostras vegetais e isolamento dos fungos}

Para o isolamento de micro-organismos endofíticos a superfície do material vegetal foi desinfestada para retirada de micro-organismos epifíticos e contaminantes. As amostras vegetais foram desinfestadas sequencialmente com etanol 70\% (30 segundos), hipoclorito de sódio 1,5\% (3 minutos), etanol 70\% (30 segundos) e enxaguadas duas vezes com água destilada esterilizada.

Quatro fragmentos de folhas, de $1 \mathrm{~cm}^{2}$ cada, foram distribuídos em placas de Petri contendo meio BDA acrescido de sulfato de estreptomicina. Para cada espécie vegetal foram feitas 3 repetições. As placas foram incubadas a $28{ }^{\circ} \mathrm{C}$ até o crescimento dos fungos serem visíveis a olho nu. Uma porção de cada micélio, morfologicamente distinto, foi repicado para outras placas contendo o mesmo meio de cultura na medida que iam surgindo nas bordas dos fragmentos vegetais durante o período de incubação. Este procedimento foi repetido até se conseguir colônias axênicas de cada isolado. Cada colônia então foi armazenada em tubos de ensaio com meio inclinado a $4{ }^{\circ} \mathrm{C}$ e em água destilada esterilizada pelo método Castellani (Dhingra \& Sinclair, 1995).

\section{3- Identificação de fungos endofíticos}

A identificação dos fungos foi realizada por métodos tradicionais onde foram consideradas as características culturais e morfológicas dos esporos, micélios e colônia, bem como aquelas observadas ao microscópio estereoscópico (Verma et al., 2009). Para visualização das estruturas microscópicas, os micélios foram fixados em lâminas de vidro, visualizados ao microscópio óptico e fotografados. Os isolados foram agrupados e os representantes de cada grupo foram selecionados para identificação por técnicas morfológicas.

\section{3- DESENVOLVIMENTO}

Dos estudos realizados até então, sobre as vegetações de Restingas no Norte Fluminense, ainda não foram relatadas a diversidade de fungos nesses ambientes, principalmente aqueles endofíticos. Das 34 espécies vegetais amostradas, foi possível obter e identificar 400 isolados de fungos (Tabela 1). Cada isolado encontra-se devidamente armazenado na micoteca do LAQUIBIO, possibilitando a repicagem e cultivo a qualquer momento, em função do interesse na utilização destes fungos. Colônias dos mesmos poderão ser fornecidos a pesquisadores mediante solicitação ao Centro de Pesquisas e Pós Graduação do ISECENSA.

O quantitativo de espécies vegetais identificadas nas restingas tem crescido e hoje estima-se que 1500 espécies estejam bem adaptadas à esse bioma. A beleza e exuberância, às vezes discretas, destas plantas sob a influência fluviomarinha, tornam-nas verdadeiros repositórios da biodiversidade. A Figura 2 ilustra a presença de alguns desses exemplares.

Essa diversidade também pode ser representada pelos fungos endofíticos encontrados quando comparados ao número de espécies vegetais estudadas, ou seja uma média de 14 isolados de fungos foram recuperados de cada espécie vegetal. A variabilidade morfológica entre as colônias é expressiva (Figura 3) e provavelmente novas espécies serão descritas, garantidas pela manutenção da coleção micológica do LAQUIBIO. 
Tabela 1. Algumas das espécies vegetais amostradas no Parque Nacional da Restinga de Jurubatiba/RJ, para isolamento de fungos endofíticos

\section{ESPÉCIE VEGETAL}

NOME CIENTÍFICO
NOME VULGAR

\section{FUNGOS}

$\mathrm{N}^{\mathrm{o}}$ de isolados obtidos

\begin{tabular}{|c|c|c|}
\hline Blutaparon portulacoides & Pirrixiu & \\
\hline Commelina erecta & Erva de Santa Luzia & \\
\hline Ipomoea pes-caprae & Batateira-da-praia & \\
\hline Schinus terebinthifolius & Aroeira & \\
\hline Allagoptera arenaria & Gurirí & \\
\hline Smilax rufescens & Aricanga & \\
\hline Byrsonima sericea & Murici & \\
\hline Sideroxylon obtusifolium & Quixaba de porco & \\
\hline Guapira opposita & Maria Mole & \\
\hline Clusia hilariana & Abaneiro & \\
\hline Eugenia astringens & Aperta & \\
\hline Remirea maritima & Pinheirinho da praia & \\
\hline Inga maritima & Ingá marítimo & \\
\hline Eugenia uniflora & Pitanga & 400 \\
\hline Erythroxylum ovalifolium & Arco de pipa & \\
\hline Myrsine parvifolia & Capororoquinha & \\
\hline Manilkara subsericea & Maçaranduba & \\
\hline Cupania emarginata & Fruto de Guaxo & \\
\hline Tocoyena bullata & Jenipabinho & \\
\hline Ipomoea imperati & Salsa da praia & \\
\hline Centrosema virginianum & Feijão-bravo & \\
\hline Capparis flexuosa & Juramento & \\
\hline Jacquinia brasiliensis & Pimenta da praia & \\
\hline Inga laurina & Ingá mirim & \\
\hline Myrsine guianensis & Capororoca de folha larga & \\
\hline Protium heptaphyllum & Almescla & \\
\hline Canavalia rosea & Feijão-de-porco & \\
\hline
\end{tabular}




\section{PR (SEVIC

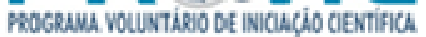
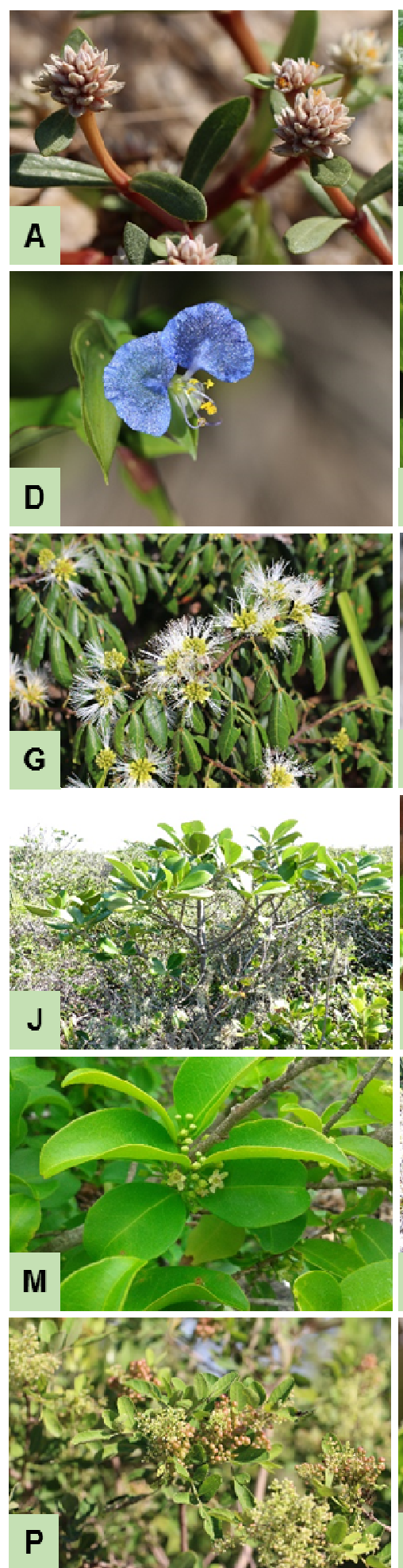

CENTRO EDLCACIONAL NOSSA SENHORA ALXILIADORA

INSTITUTOS SUPERIORES DE ENSINO DO CENSA CENTRO DE PESQUISA E PÓS-GRADUAÇ̃O
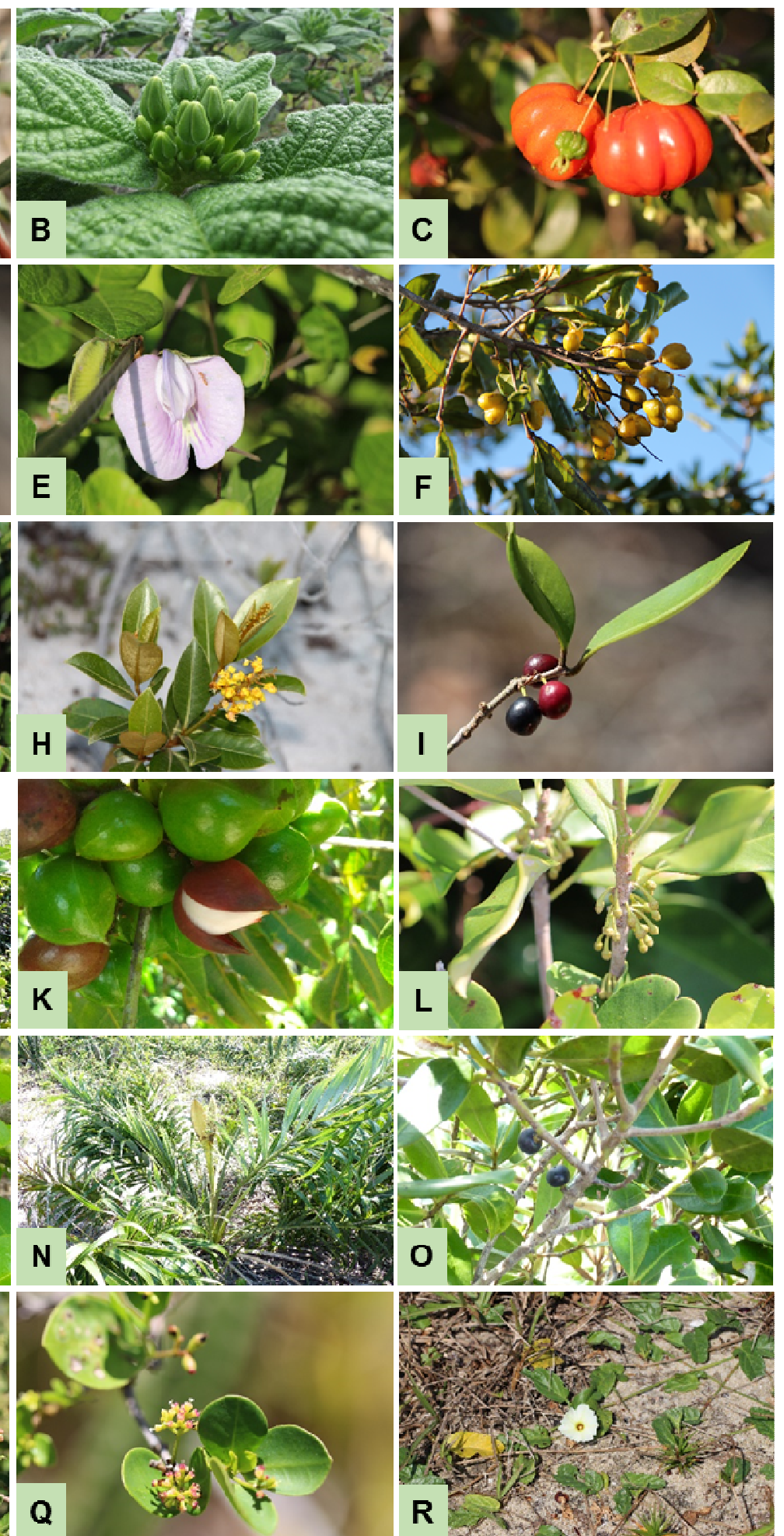

Figura 2. Espécies vegetais do Parque Nacional da Restinga de Jurubatiba, RJ. A) Blutaparon portulacoides; B) Tocoyena bullata; C) Eugenia uniflora; D) Commelina erecta; E) Centrosema virginianum; F) Cupania emarginata; G) Inga maritima; H) Byrsonima sericea; I) Eugenia astringens; J) Clusia hilariana; K) Protium heptaphyllum; L) Manilkara subsericea; M) Erythroxylum ovalifolium; N) Allagoptera arenaria; O) Myrsine parvifolia; P) Schinus terebinthifolius; Q) Guapira opposita e R) Ipomoea imperati. 


\section{PR (⿶SVIC}

CENTRO EDUCACIONAL NOSSA SENHORA AUXILIADORA

INSTITUTOS SUPERIORES DE ENSINO DO CENSA

CENTRO DE PESQUISA E PÓS-GRADUAÇ̃̃o
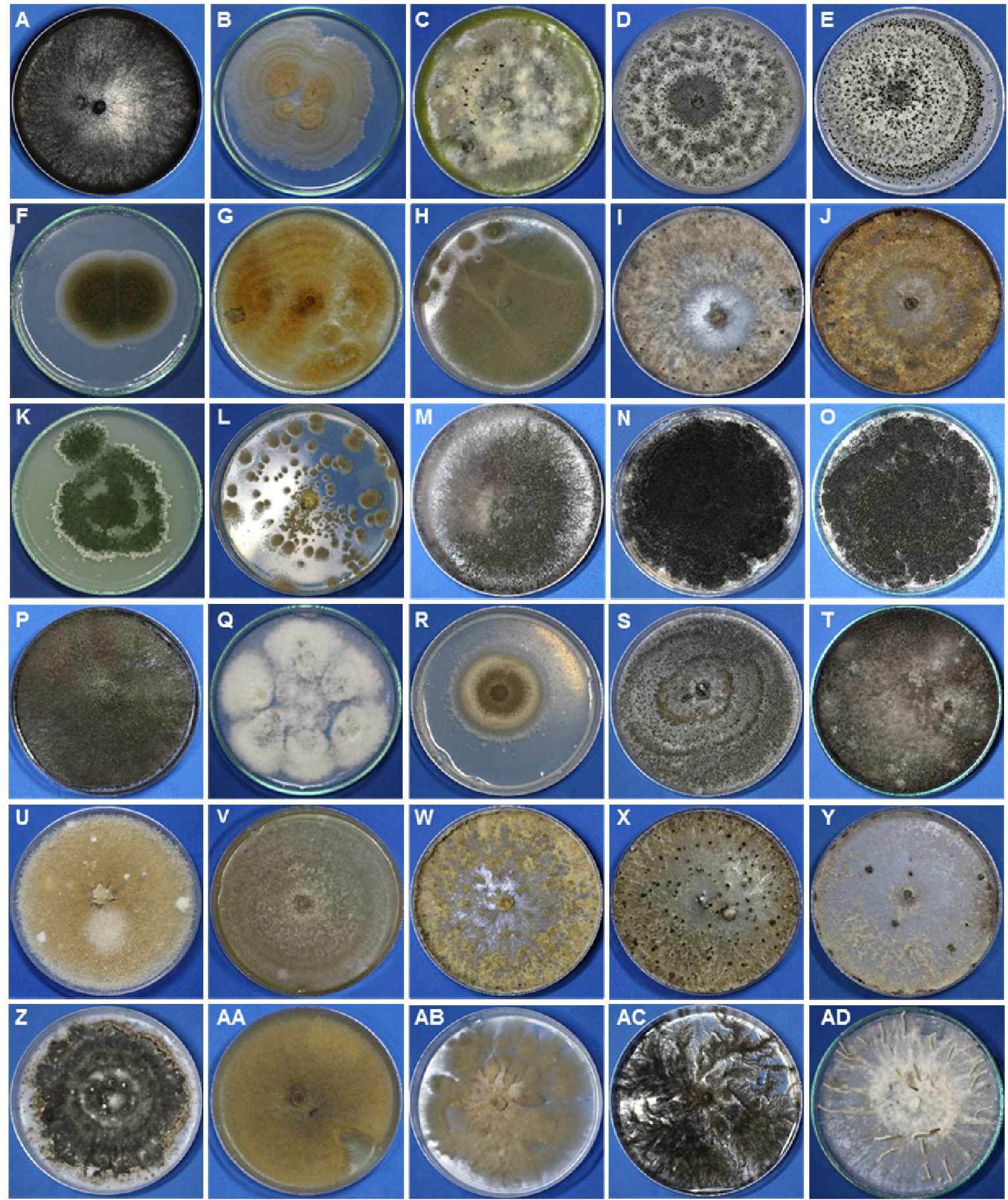

Figura 3. Colônias de fungos endofíticos obtidas de amostras de espécies vegetais do Parque Nacional da Restinga de Jurubatiba, RJ. Gêneros: A) Exserohilum; B) Gliocladium; C, D e E) Pestalotiopsis; F) Cladosporium; G) Curvularia; H) Penicillium; I e J) Phoma; K) Trichoderma; L) Aspergillus; M) Bipolaris; N, O e P) Guignardia; Q) Beauveria; R) Alternaria; S) Colletotrichum; T) Nigrospora; U e V) Fusarium; W, X e Y) Phomopsis; Z, AA, AB, AC e AD) isolados não esporulados. 


\section{CENTRO EDUCACIONAL NOSSA SENHORA AUXILIADORA \\ INSTITUTOS SUPERIORES DE ENSINO DO CENSA \\ CENTRO DE PESQUISA E PÓS-GRADUAÇ̃̃o}

\section{4- CONSIDERAÇÕES FINAIS}

Embora tenha havido um crescente aumento nos trabalhos de detecção de fungos endofíticos nos últimos anos, praticamente nada se conhece a respeito da presença e identificação dos mesmos em plantas de restinga. Isso representa uma fonte potencial de diversidade de espécies e aguça a necessidade de se conhecer sua presença, relações e aplicabilidades, uma vez que as restingas são consideradas espaços ímpares, pois convergem para ela biomas de Mata Atlântica, Cerrado e Caatinga, tornando-a um ambiente totalmente diferente para a prospecção de novas espécies fúngicas.

\section{5- REFERÊNCIAS}

Araujo, D.S.D., Scarano, F.R., Sá, C.F.C., Kurtz, B.C., Zaluar, H.L.T., Montezuma, R.C.M. \& Oliveira, R.C. (1998). Comunidades vegetais do Parque Nacional da Restinga de Jurubatiba. In: Ecologia das lagoas costeiras do Parque Nacional da Restinga de Jurubatiba e do Município de Macaé (RJ) (F.A. Esteves, ed.). Universidade Federal do Rio de Janeiro, Rio de Janeiro, p.39-62.

Campos, Rômulo. Parque Nacional da Restinga de Jurubatiba (2015). 1. ed. Macaé/RJ: Íris Mídia, v.1. 195p.

Cogliatti-Carvalho,L., Freitas, A.F.N., Rocha, C. F.D., Van Sluys, M. Variação na estrutura e na composição de Bromeliaceae em cinco zonas de restinga no Parque Nacional da Restinga de Jurubatiba, Macaé, RJ. 1. Revta brasil. Bot., São Paulo, V.24, n.1, p.1-9, mar. 2001

Dhingra, O.D., Sinclair, J.B. Basic plant pathology methods. 2 ed. CRC Press. 434p. 1995.

Freire, M.G.M., Mussi-Dias, V., Siqueira, G.M.B., Nascimento, D.F. (2015). O Tempo e a Restinga/Time and Restinga. 1. ed. Rio de Janeiro: RR Donnelley, v.1. 349p.

Gunatilaka, A.A.L. Natural products from plant-associated microorganisms: distribution, structural diversity, bioactivity and implication of their occurence. J. Nat. Prod., 69: 509-526. 2006.

Imbassahy, A.A.A., Costa, D. P. D., Araujo, S. D. Briófitas do Parque Nacional da Restinga de Jurubatiba, RJ, Brasil. Acta bot. bras. 23(2): 558-570. 2009.

Luz, J. L., Mangolin, R., Esbérard, C. E. L., Bergallo, H. G. Morcegos (Chiroptera) capturados em lagoas do Parque Nacional da Restinga de Jurubatiba, Rio de Janeiro, Brasil. Biota Neotrop., vol. 11, no. 4, 2011.

Reis, R. C. C. Palmeiras (Arecaceae) das Restingas do Estado do Rio de Janeiro, Brasil, Acta bot. bras. 20(3): 501-512. 2006.

Strobel, G., Daisy, B., Castillo, U., Harper, J. Natural products from endophytic microorganisms. J. Nat. Prod., 67: 257-268. 2004.

Vainer, A.G. Conflitos ambientais em evidência na criação e manejo de um parque nacional: o caso do Parque Nacional da Restinga de Jurubatiba. Trabalho publicado nos Anais do XIX Encontro Nacional do CONPEDI, Fortaleza/CE, de 9 a 12 de Junho de 2010.

Verma, V.C., Gond, S.K., Kumar, A., Mishra, A., Kharwar, R.N., Gange, A.C. Endophytic Actinomycetes from Azadirachta indica A. Juss.: isolation, diversity, and antimicrobial activity. Microb Ecol, 57: 749-756. 2009. 